\title{
Large Aperture Micro-Focus KB Mirrors for Spectroscopy Experiments at the Advanced Light Source
}

\author{
T. Warwick ${ }^{1}$, N. Andresen ${ }^{1}$, J. Comins ${ }^{1}$, A. Franck ${ }^{1}$, M. Gilles ${ }^{1}$, T. Tonnessen ${ }^{2}$ and \\ T. Tyliszczak ${ }^{1}$ \\ ${ }^{1}$ Lawrence Berkeley National Laboratory, Berkeley, California 94720, USA \\ ${ }^{2}$ InSync Inc., Albuquerque, New Mexico 87107, USA
}

\begin{abstract}
General purpose refocus mirrors using Kirkpatrick-Baez geometry have been designed, built and installed at a new undulator beam-line facility to provide spot sizes smaller than 10 microns for specialized spectroscopy experiments at the Advanced Light Source. All the available flux is focused and the focal length is adjustable. The mirrors are fully computer controlled and can be detuned to create a spot as big as 500 microns.
\end{abstract}

\section{INTRODUCTION}

The Molecular Environmental Science (MES) beam-line 11.0.2 at the ALS illuminates electron and photon spectroscopy experiments that measure sample surfaces in the presence of water. Small apertures and windows are employed to retain the water vapor [1]. Small x-ray spots are required to pass the illuminating radiation through these holes. An entrance slit-less emission spectrometer is under development [2] that will collect emitted x-rays most efficiently. This instrument relies on a small illumination size (7 microns) in the dispersive direction to achieve its design spectral resolution. These two requirements for small spots have been met by the construction of automated KB mirrors [3], focusing the undulator beam to sizes less than 10 microns. The bending and steering is computer controlled and accessible to the experimenter. The mirrors operate with 3 degrees deflection and the bending is severe. The optics are made of metal, so that the bending hardware can be attached without glue, for clean operation in UHV. The substrates are relatively thin $(20 \mathrm{~mm})$, to limit the bending forces and to allow significant tuning range, including a defocused setting. The width of the substrate $(\approx 50 \mathrm{~mm})$ is profiled, to generate an elliptical surface with two-couple bending. One of the two mirrors deflects vertically and is subject to deformation under gravity, so the substrate width-profile includes a gravity-sag correction. After bending, the measured residual slope errors are about 1 micro-radian r.m.s. and x-ray spot sizes of 7 microns FWHM have been achieved at a working distance of 1.4 meters. This design concept originates in the work of Howells et. al. [4].

An alternative KB design has been reported [5,6] with similar properties. In this design the substrate is thick enough to avoid gravity sag, and is polished close to an ellipse, with a small amount of bender adjustment.

\section{SUBSTRATE DESIGN}

Typically, a design begins with a specified object distance (u), image distance (v), grazing angle $(\theta)$ and the ellipse parameters follow. Semi-major-axes are a and b, e is the ellipticity. The ellipse is highly eccentric and a coordinate system is adopted so that its focii are on the horizontal (x) axis.

$$
\mathrm{a}=(\mathrm{u}+\mathrm{v}) / 2 \quad e=\left\{\frac{u^{2}+v^{2}+2 u v \cos (2 \theta)}{(u+v)^{2}}\right\}^{\frac{1}{2}} \quad \mathrm{~b}^{2}=a^{2}\left(1-\mathrm{e}^{2}\right)
$$


We wish to work with a coordinate (p) that corresponds to the length along the mirror. The following formula is adopted to map a point on the mirror to a point on the ellipse:

$$
x(\mathrm{p}) \approx p\left\{\frac{1+\left(x_{0}^{2} b^{2} / a^{2}\right)}{a^{2}-x_{0}{ }^{2}}\right\}^{-\frac{1}{2}}+x_{0}
$$

In this approximation, $\mathrm{p}$ is the distance along the tangent to the ellipse through the mirror at $\left(\mathrm{x}_{0}, \mathrm{y}_{0}\right)$ from $\left(\mathrm{x}_{0}, \mathrm{y}_{0}\right)$ to the point on the tangent with coordinate $\mathrm{x}(\mathrm{p})$. The point on the ellipse with coordinate $\mathrm{x}(\mathrm{p})$ lies close to the point on the tangent with coordinate $x(p)$ provided the ellipse is highly eccentric $(a) b$, which is true for small $\theta$ ).

The curvature of the elliptical surface is:

$$
\frac{1}{\rho_{\text {ellipse }}}=\frac{d^{2} y}{d x^{2}}\left\{1+\frac{d y^{2}}{d x}\right\}^{-\frac{3}{2}}= \pm a b\left\{a^{2}-x(p)^{2}+\frac{x(p)^{2} b^{2}}{a^{2}}\right\}^{-\frac{3}{2}}
$$

When designing an elliptical mirror, the curvature of the mirror is matched to the curvature of the required ellipse. For a uniform beam bent with independent couples at each end it is possible to match the curvature at two points along the length.

A mirror with a tailored profile can be arranged to have the correct curvature everywhere, even under the influence of gravity. It then remains to position and tilt the curved surface correctly to form the image at the desired location.

\section{Bent Beams}

A beam of width $\mathrm{w}$ and thickness $\mathrm{t}$ with density $\mathrm{d}$ experiencing the effects of gravity and bent with a different couple applied at each end has a bending moment (M) which is a function of the length (p) along the mirror.

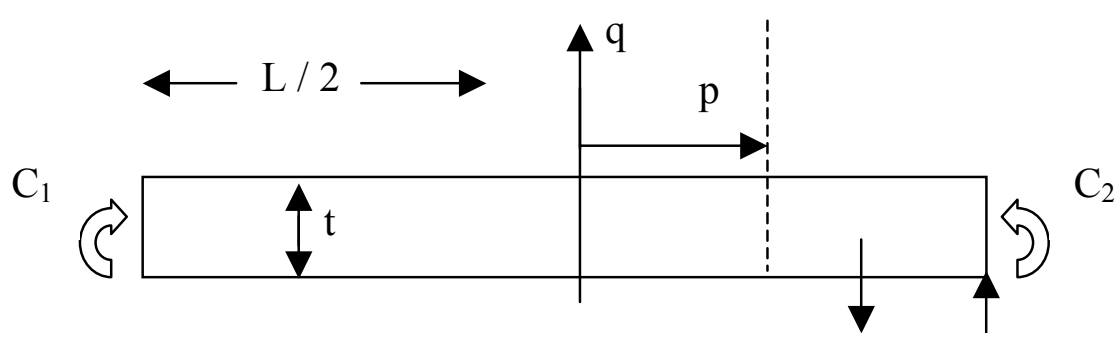

$\mathrm{dw} \operatorname{tg}(\mathrm{L} / 2-\mathrm{p}) \quad \mathrm{d} w \mathrm{tg}(\mathrm{L} / 2)$

$$
M(p)=\left\{C_{1}(L / 2-p)+C_{2}(L / 2+p)\right\} / L+(d w \operatorname{tg} / 2)(L / 2-p)(L / 2+p)
$$

Beam theory gives: $\frac{d^{2} q}{d p^{2}}=M(p) / E I$ where E is Young's modulus and I is the section moment of inertia. For a rectangular section of thickness $\mathrm{t}$ and width $\mathrm{w}: I=w t^{3} / 12$ The curvature of the (approximately horizontal) surface is:

$$
\frac{1}{\rho_{\text {bender }}}=\frac{d^{2} q}{d p^{2}}\left\{1+\frac{d q^{2}}{d p}\right\}^{-\frac{3}{2}} \approx \frac{d^{2} q}{d p^{2}}
$$

Now the curvature of the bent beam can be matched to that of the required ellipse. The computational procedure is as follows. First the couples are tuned using a beam with constant section, with gravity if appropriate, to give 
curvature matched at two points near each end. Then a free variation of the width $w(p)$ is allowed along the mirror to match the curvature along the entire length, with gravity effects included.

$$
w(p) \approx\left(12 \rho_{\text {ellipse }} / E t^{3}\right) M(p)
$$

Iteration is required to include gravity properly, using the previously computed width-profile to provide the gravity effects for the next computation of the width. Errors are negligible after two iterations.

\section{MECHANICAL DESIGN}

A working distance for these mirrors is established to allow for interchangeability of experiments on a rotating platform and to provide space for differential pumping as required. The last KB mirror focuses vertically with a $3^{\circ}$ downward deflection at a distance of $1.45 \mathrm{~m}$. This defines the demagnification from the monochromator exit aperture which is $7.8 \mathrm{~m}$ upstream, typically tens of microns square. The two KB mirrors are $0.8 \mathrm{~m}$ apart, and $350 \mathrm{~mm}$ long. There are two spring arms at each end of the optic. One is a pre-load-couple, set with a turnbuckle. The second spring is pulled by the motorized linear feedthrough that can tune and de-tune the curvature. The bender mechanism is flange-mounted. A monolithic aluminum vacuum chamber adds rigidity and carries both mirrors. The chamber has transverse dimensions of $150 \mathrm{~mm}$. This narrow chamber saves space near the experiments. Positioning of the mirrors and small adjustments of the deflection angle (for steering under computer control) are achieved

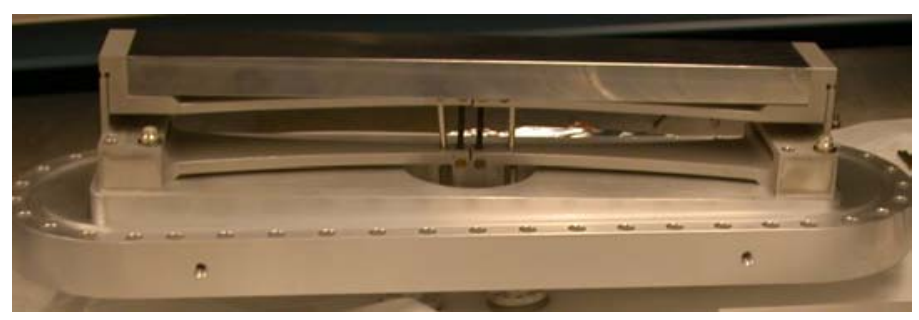

FIGURE 1. Flange mounted bender with dummy substrate. by moving the chamber.

\section{BENCH TUNING AND METROLOGY}

These mirrors are tuned under the Long Trace Profiler [7] at the ALS. Because of the gravity sag compensation the LTP measurements must be made in the operational orientation. The design elliptical shape is achieved with residual slope errors of approximately $1.5 \mu \mathrm{rad}$ r.m.s. over the central $200 \mathrm{~mm}$ of the optic. The tuning is laborious. We found that some retuning was necessary after installation. However the bench tuning is essential to verify the substrate design and to set the travel range of the motorized bending to include the design shape. Switches are set to limit the travel and one of these is used as a homing reference.

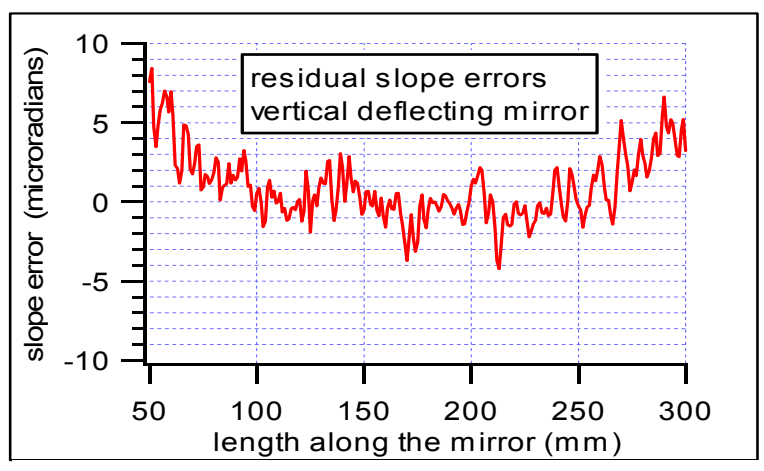

FIGURE 2. Slope errors after bench tuning.

\section{PERFORMANCE WITH X-RAYS}

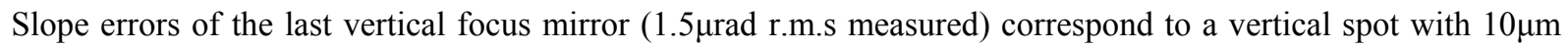
FWHM. After retuning the mirrors to minimize the measured x-ray spot size and given that the beam actually fills only $100 \mathrm{~mm}$ of the mirror (at $400 \mathrm{eV}$ ), we observe a better result. The FWHM measured is between 6 and $7 \mu \mathrm{m}$. The exit slit aperture is $10 \mu \mathrm{m}$ square for these spot size measurements, and demagnified to $1.8 \mu \mathrm{m}$ at the focus. The measurements were made by capturing images of the x-rays focused on a YAP crystal, using a visible light microscope with a spatial resolution of about 3 microns. The measured vertical size meets the requirements of the vertically-dispersing entrance-slit-less emission spectrometer which is under development. The horizontal de- 
magnification is less than the vertical, and under typical operational conditions the horizontal aperture at the exit slit would be wider, to increase the flux.
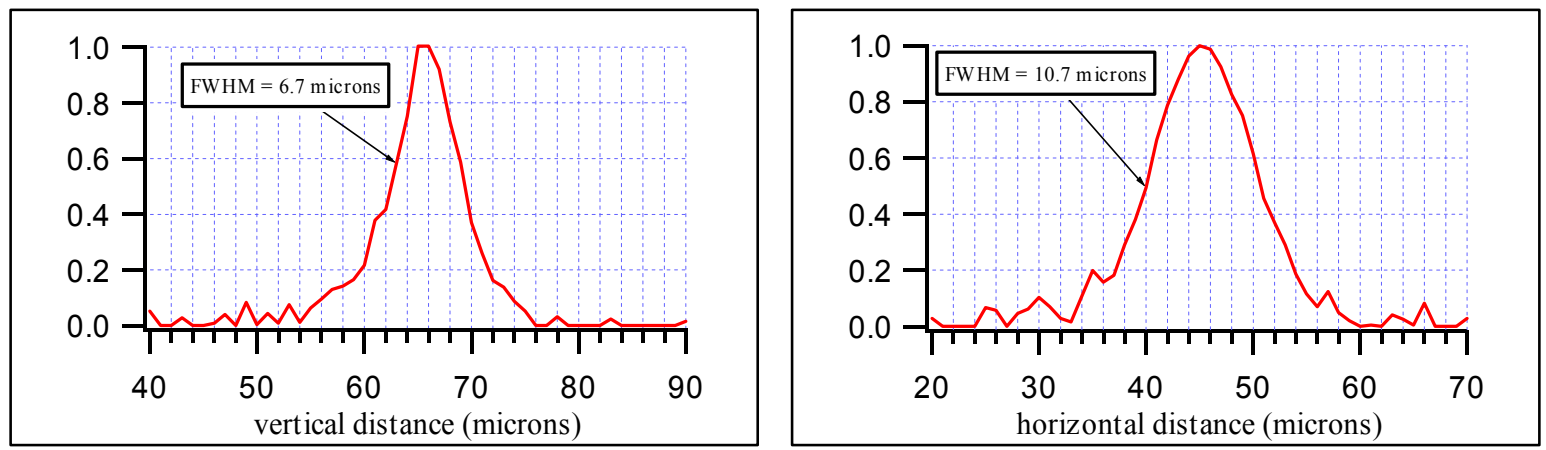

FIGURE 3. Measured x-ray spot profiles at the focus.

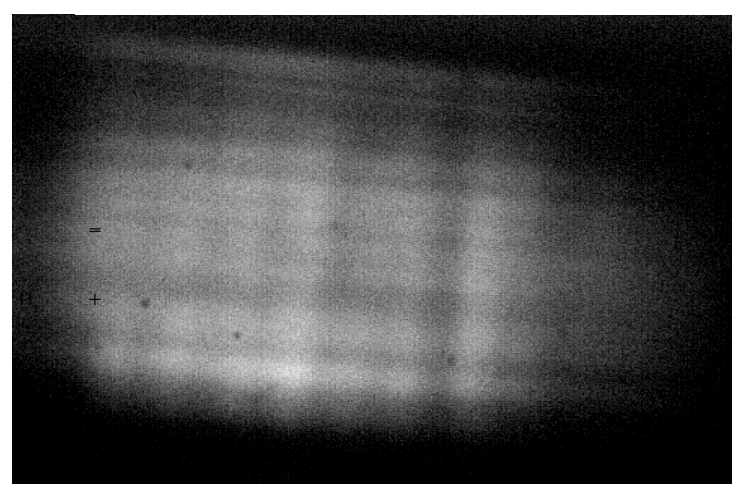

The focusing is adjustable and the small spot can be moved $0.5 \mathrm{~m}$ down-stream without appreciable enlargement, by re-tuning the benders. (If the benders are not changed the divergence downstream of the focus is approximately $3 \mathrm{mrad}$ FWHM). Additionally a defocused beam $500 \mu \mathrm{m}$ in diameter can be generated by completely de-tuning the benders. However, under these conditions the slope errors from polishing show as streaked intensity variations across the illumination field. Tuned focusing can be recovered under computer control.

FIGURE 4. Defocused spot $500 \mu \mathrm{m}$ diameter.

This project would not have been possible without the expertise of Don McGill and the vacuum group at the ALS. The Advanced Light Source and this work are supported by the Director, Office of Science, Office of Basic Energy Sciences, Division of Materials Sciences and the Division of Chemical Sciences, Geosciences, and Biosciences of the U.S. Department of Energy at Lawrence Berkeley National Laboratory under Contract No. DEAC03-76SF00098.

\section{REFERENCES}

1. Mun, B. S., Lebedev, G., Requejo, F., Liu, H., Ogletree, D. F., Salmeron, M., Wong, E., Wingert, M., Hussain, Z., and Ross, P. N. Jr., "The development of ambient pressure X-ray photoelectron spectroscopy (XPS) chamber" Proc. Eighth International Conf. Synchrotron Radn, Intrum., American Institute Physics, 2003, to be published.

2. Underwood, J, Hussain, Z., McKinney, W.R. and Warwick, T.

“1.5 Meter VLS Emission Spectrometer", ALS Beamline Note, LSBL 622, 29 March, 2002.

3. Kirkpatrick, P. and Baez, A.V., "Formation of Optical Images by Xrays" J. Opt.Soc.Am. 38, 1948, pp. 776-774.

4. Howells, M.R., Cambie, D., Duarte, R.M., Irick, S., MacDowell, A., Padmore, H.A., Renner, T., Rah, S. and Sandler, R., "Theory and practice of elliptically bent X-ray mirrors", Opt. Eng. 39, 2000, pp.2748-2762

5. Ferme, J.J., “An advanced KB mirror pair for microfocusing” Nucl. Instrum. Meth. A467-468, 2001, pp. 279282.

6. Cocco, D., Marsi, M., Kiskinova, M., Prince, K.C., Schmidt, T., Heun, S., and Bauer, E., “A Microfocusing VLS grating based beamline for advanced microscopy" EUV, Xray and Neutron Optics and Sources", Proc. SPIE 3767, 1999, pp.271-279

7. Locklin, S., Rah, S., Irick, S., Howells, M., "Progress in elliptical bender mirror adjustment using the Long Trace Profiler," ALS Beamline Note, LSBL 381, 26 May, 1997. 